\title{
Effectiveness of acyclovir in the treatment of pityriasis rosea. A systematic review and meta-analysis ${ }^{*}$
}

\author{
Milton Rodriguez-Zuniga ${ }^{1}$, Natalie Torres ${ }^{2}$, Herney Garcia-Perdomo ${ }^{3}$
}

DOI: http:/ /dx.doi.org/10.1590/abd1806-4841.20187252

\begin{abstract}
BACKGROUND: There is a lack of evidence to support acyclovir administration in pityriasis rosea.
Oвјестіv: To determine the efficacy of acyclovir in patients with typical pityriasis rosea.

METHODS: A systematic review and meta-analysis of experimental studies was performed in MEDLINE, SCOPUS, EMBASE and others, from January 1990 to October 2016 on acyclovir for pityriasis rosea. Random effect model was used to find the pooled Risk Ratio. Outcomes, evaluated between weeks 1 to 8, were regression of lesions, cessation of lesions, decrease of symptoms and duration of disease. Comparisons were acyclovir vs. placebo; acyclovir vs. symptomatic treatment; acyclovir vs. antibiotic; acyclovir vs. observation and combined therapy (acyclovir plus symptomatic treatment) vs. symptomatic treatment alone.

RESULTS: Seven papers were analyzed with 324 participants, of which 159 received acyclovir and 165 were controls. Acyclovir was superior to placebo for complete regression of lesions at week 1 (Risk Ratio 5.72, CI95\% 2.36-13.88). However, combined therapy was not superior to symptomatic treatment at week 4 (Risk Ratio 1.46, CI95\% 0.93-2.29). Individual studies showed the superiority of acyclovir for the control of symptoms and pruritus.

STUDY LIMITATIONS: We faced differences designs of trials and inconsistency between reports.

CONCLUSION: Symptomatic treatment is a reasonable option for pityriasis rosea, and the addition of acyclovir is justified for the control of symptoms and pruritus.
\end{abstract}

Keywords: Acyclovir; Histamine antagonists; Meta-analysis as topic; Pityriasis rosea; Pruritus

\section{INTRODUCTION}

Pityriasis rosea (PR) is an acute inflammatory skin disease that occurs worldwide, more often in children and young people. ${ }^{1,2}$ Typically, an exanthema of smaller patches develops across the trunk, that usually lasts 8 weeks, with moderate to severe pruritus. 3,4 The etiology is still unclear, but many features suggest a viral infectious cause: at least half of patients reports symptoms compatible with a viral upper respiratory infection, the early age of onset, the self-limited clinical course and the low rate of relapses. ${ }^{5,6}$ Accordingly, several authors have found the presence of Human Herpes Virus type 6 and 7 (HHV-6 and HHV-7) in patients with PR, however, the evidence is not enough to confirm a direct association. ${ }^{4,7,8}$

Current treatments consist on topical and systemic therapy, mainly as symptomatic drugs, as PR is a self-limited disease. ${ }^{9}$ Yet, Drago et al, ${ }^{10}$ first described the efficacy of acyclovir for PR with

\footnotetext{
Received 28 April 2017.

Accepted 20 September 2017.

* Work conducted at the Universidad Nacional Mayor de San Marcos, Lima, Peru.

Financial support: None.

Conflict of interest: None.

Dermatology Department, Hospital Nacional Daniel Alcides Carrion, Universidad Nacional Mayor de San Marcos, Lima, Peru.

Dermatology Department, Hospital Nacional Dos de Mayo, Universidad Nacional Mayor de San Marcos, Lima, Peru.

Postgraduate Clinical Programs, Universidad del Valle, Cali, Colombia.
} 


\begin{tabular}{|c|c|c|c|}
\hline Section/topic & \# & Checklist item & Reported on page \# \\
\hline \multicolumn{4}{|l|}{ TITLE } \\
\hline Title & 1 & Identify the report as a systematic review, meta-analysis, or both & 1 \\
\hline \multicolumn{4}{|l|}{ ABSTRACT } \\
\hline Structured summary & 2 & $\begin{array}{l}\text { Provide a structured summary including, as applicable: background; objectives; data } \\
\text { sources; study eligibility criteria, participants, and interventions; study appraisal and } \\
\text { synthesis methods; results; limitations; conclusions and implications of key findings; } \\
\text { systematic review registration number }\end{array}$ & 22 \\
\hline \multicolumn{4}{|l|}{ INTRODUCTION } \\
\hline Rationale & 3 & Describe the rationale for the review in the context of what is already known & 4 \\
\hline Objectives & 4 & $\begin{array}{l}\text { Provide an explicit statement of questions being addressed with reference to participants, } \\
\text { interventions, comparisons, outcomes, and study design (PICOS) }\end{array}$ & 4 \\
\hline \multicolumn{4}{|l|}{ METHODS } \\
\hline Protocol and registration & 5 & $\begin{array}{l}\text { Indicate if a review protocol exists, if and where it can be accessed (e.g., Web address), } \\
\text { and, if available, provide registration information including registration number }\end{array}$ & 4 \\
\hline Eligibility criteria & 6 & $\begin{array}{l}\text { Specify study characteristics (e.g., PICOS, length of follow-up) and report characteristics } \\
\text { (e.g., years considered, language, publication status) used as criteria for eligibility, giving } \\
\text { rationale }\end{array}$ & 5 \\
\hline Information sources & 7 & $\begin{array}{l}\text { Describe all information sources (e.g., databases with dates of coverage, contact with } \\
\text { study authors to identify additional studies) in the search and date last searched }\end{array}$ & 4 \\
\hline Search & 8 & $\begin{array}{l}\text { Present full electronic search strategy for at least one database, including any limits used, } \\
\text { such that it could be repeated }\end{array}$ & 4 \\
\hline Study selection & 9 & $\begin{array}{l}\text { State the process for selecting studies (i.e., screening, eligibility, included in systematic } \\
\text { review, and, if applicable, included in the meta-analysis) }\end{array}$ & 5 \\
\hline Data collection process & 10 & $\begin{array}{l}\text { Describe method of data extraction from reports (e.g., piloted forms, independently, in } \\
\text { duplicate) and any processes for obtaining and confirming data from investigators }\end{array}$ & 5 \\
\hline Data items & 11 & $\begin{array}{l}\text { List and define all variables for which data were sought (e.g., PICOS, funding sources) } \\
\text { and any assumptions and simplifications made }\end{array}$ & 5 \\
\hline Risk of bias in individual studies & 12 & $\begin{array}{l}\text { Describe methods used for assessing risk of bias of individual studies (including specifi- } \\
\text { cation of whether this was done at the study or outcome level), and how this information } \\
\text { is to be used in any data synthesis }\end{array}$ & 6 \\
\hline Summary measures & 13 & State the principal summary measures (e.g., risk ratio, difference in means) & 6 \\
\hline Synthesis of results & 14 & $\begin{array}{l}\text { Describe the methods of handling data and combining results of studies, if done, includ- } \\
\left.\text { ing measures of consistency (e.g., } I^{2}\right) \text { for each meta-analysis }\end{array}$ & 6 \\
\hline Risk of bias across studies & 15 & $\begin{array}{l}\text { Specify any assessment of risk of bias that may affect the cumulative evidence (e.g., publi- } \\
\text { cation bias, selective reporting within studies) }\end{array}$ & 6 \\
\hline Additional analyses & 16 & $\begin{array}{l}\text { Describe methods of additional analyses (e.g., sensitivity or subgroup analyses, meta-re- } \\
\text { gression), if done, indicating which were pre-specified }\end{array}$ & 6 \\
\hline \multicolumn{4}{|l|}{ RESULTS } \\
\hline Study selection & 17 & $\begin{array}{l}\text { Give numbers of studies screened, assessed for eligibility, and included in the review, } \\
\text { with reasons for exclusions at each stage, ideally with a flow diagram }\end{array}$ & 6 \\
\hline Study characteristics & 18 & $\begin{array}{l}\text { For each study, present characteristics for which data were extracted (e.g., study size, } \\
\text { PICOS, follow-up period) and provide the citations }\end{array}$ & 6, Table1 \\
\hline Risk of bias within studies & 19 & $\begin{array}{l}\text { Present data on risk of bias of each study and, if available, any outcome level assessment } \\
\text { (see item 12) }\end{array}$ & Fig. 7 \\
\hline Results of individual studies & 20 & $\begin{array}{l}\text { For all outcomes considered (benefits or harms), present, for each study: (a) simple } \\
\text { summary data for each intervention group (b) effect estimates and confidence intervals, } \\
\text { ideally with a forest plot }\end{array}$ & Table 1 \\
\hline Synthesis of results & 21 & $\begin{array}{l}\text { Present results of each meta-analysis done, including confidence intervals and measures } \\
\text { of consistency }\end{array}$ & 7 \\
\hline Risk of bias across studies & 22 & Present results of any assessment of risk of bias across studies (see Item 15). & Fig. 7 \\
\hline Additional analysis & 23 & $\begin{array}{l}\text { Give results of additional analyses, if done (e.g., sensitivity or subgroup analyses, me- } \\
\text { ta-regression [see Item 16]) }\end{array}$ & 8 \\
\hline \multicolumn{4}{|l|}{ DISCUSSION } \\
\hline Summary of evidence & 24 & $\begin{array}{l}\text { Summarize the main findings including the strength of evidence for each main outcome; } \\
\text { consider their relevance to key groups (e.g., healthcare providers, users, and policy makers) }\end{array}$ & 8,9 \\
\hline Limitations & 25 & $\begin{array}{l}\text { Discuss limitations at study and outcome level (e.g., risk of bias), and at review-level (e.g., } \\
\text { incomplete retrieval of identified research, reporting bias) }\end{array}$ & 11 \\
\hline Conclusions & 26 & $\begin{array}{l}\text { Provide a general interpretation of the results in the context of other evidence, and impli- } \\
\text { cations for future research }\end{array}$ & $11-12$ \\
\hline \multicolumn{4}{|l|}{ FUNDING } \\
\hline Funding & 27 & $\begin{array}{l}\text { Describe sources of funding for the systematic review and other support (e.g., supply of } \\
\text { data); role of funders for the systematic review }\end{array}$ & 1 \\
\hline
\end{tabular}


promising results. Nowadays, there is still a lack of high quality evidence to support acyclovir administration in PR. Recommendations suggest its use in cases of extensive, relapsing, persistent disease or during pregnancy, mostly based on expert opinions. ${ }^{11}$ We aimed to determine the efficacy of acyclovir in comparison to other interventions in patients with typical PR, both adults and children.

\section{METHODS}

This systematic review and meta-analysis was performed according to the Cochrane Collaboration recommendations and the PRISMA guidelines (Chart 1). ${ }^{12-14}$ Protocol was registered on PROSPERO (CRD42016049318).

\section{Search Strategy}

A database search was independently conducted by two reviewers (MR and NT) in MEDLINE (accessed via PubMed), SCOPUS, EMBASE, SCIENCE DIRECT, LILACS and the Cochrane Central Register of Controlled Trials for the period of January 1990 until October 2016. ${ }^{15,16}$ The search strategy was specific for each database and included a combination of the Medical Subject Heading and free text terms for "Acyclovir", "Pityriasis rosea" and "Clinical tri$\mathrm{al}^{\prime}$ (Chart 2). For unpublished studies we reviewed grey literature and experts' opinion on the topic. In addition, we scanned bibliographies of published studies by a manual search of the literature; reviewed virtual libraries of universities and theses; requested authors for papers presented at conferences and congresses of dermatology societies; and contacted the pharmaceutical industry. We also searched at ClinicalTrials.gov database to find additional studies that were not included in the initial search. There are no restrictions of language to select the studies for this review.

\section{Eligibility criteria}

We included parallel-clinical trials (experimental and quasi-experimental), with open-labeled or blinded designs; studying the efficacy of acyclovir for PR. Participants included were immunocompetent and nonpregnant females and males, children and adults with PR diagnosed according to the investigator criteria and specific clinical features.

Studies should have two or more study arms, at least one arm on acyclovir (with or without symptomatic treatment), and a control group (on placebo, symptomatic treatment, antibiotics or nothing). Symptomatic treatment consists on oral or topical antihistamines, oral or topical steroids, and topical balsams. ${ }^{9,17}$

We excluded studies lacking complete data in any arm of study, after exhausting efforts to contact the authors for full data. Overlapping studies were included after discarding the one with the smaller study population.

The comparisons were (1) Acyclovir vs. placebo; (2) Acyclovir vs. symptomatic treatment; (3) Acyclovir vs. antibiotic; (4) Acyclovir vs. observation; (5) Acyclovir plus symptomatic treatment (combined therapy) vs. symptomatic treatment alone.

\section{Outcomes}

The primary outcome was the proportion of patients achieving regression of lesions after the treatment of oral acyclovir. Regres-

\section{Chart 2: Protocol search strategy}

The search strategy was specific for each database according to the medical subject headings $(\mathrm{MeSH})$ and free text terms for the key concepts. The search terms were combined as follow: "pityriasis rosea"[MeSH Terms] AND "acyclovir"[MeSH Terms] AND "clinical trial"[MeSH Terms]

\section{PUBMED}

(Pityriasis Rosea OR Pityriasis OR pityriasis rosea of gilbert OR pityriasis rosea Gilbert) AND (Acyclovir OR Acycloguanosine OR Acic* OR acyc*) No filters will be added

\section{COCHRANE}

We will search for "all text", it will be limited by "product type", indicating the point "trials". The terms will be combined as follows: (Pityriasis Rosea OR Pityriasis OR pityriasis rosea of gilbert OR pityriasis rosea Gilbert) AND (Acyclovir OR acyclovir OR Acycloguanosine OR Acic* OR acyc*)

\section{SCOPUS}

We are going to search (All words) in "All fields" (full text) for: (Pityriasis Rosea OR Pityriasis OR pityriasis rosea of gilbert OR pityriasis rosea Gibert) AND (Aciclovir OR Acycloguanosine OR Acic* OR acyc*) AND (clinical trial OR clinical trials,randomized OR controlled clinical trials, randomized)

\section{SCIENCEDIRECT}

Search results: 61 results found for pub-date $>1989$ and (Pityriasis Rosea OR Pityriasis) AND (Acyclovir OR Acycloguanosine) AND (clinical trial) AND LIMIT-TO(topics, "patient,treatment,acad dermatol,dermatology,skin,clinical")

\section{BIREME}

(Pityriasis Rosea OR Pityriasis OR pityriasis rosea of gilbert OR pityriasis rosea Gibert) AND (Aciclovir OR Acycloguanosine OR Acic* OR acyc*). All the studies that include one of the following terms in the tittle will be considered: limits: "humans" and main topic: "Pytiriasis rosea"

\section{LILACS}

(Pityriasis Rosea OR Pityriasis OR pityriasis rosea of gilbert OR pityriasis rosea Gibert) AND (Aciclovir OR Acycloguanosine OR Acic* OR acyc*) AND (clinical trial OR clinical trials,randomized OR controlled clinical trials,randomized)

\section{Other resources}

Clinicaltrials.gov:

(Pityriasis Rosea) AND (Acyclovir)

sion of lesions is defined as decrease or disappearance of erythema in all lesions leaving desquamation or pigmentation. Secondary outcomes were (i) The proportion of patients that achieved cessation of new lesions. (ii) The proportion of patients that had decreased systemic symptoms (pruritus). (iii) Time for complete clearance of lesions. Outcomes were measured at several weeks after treatment was initiated.

\section{Study selection}

Two reviewers (MR and NT) independently screened the titles and abstracts of all the retrieved articles, followed by the full 
texts of the articles considered potentially eligible for the study. They independently collected the data using a standardized data sheet. Both authors confirmed all entries and checked at least twice for completeness and accuracy. Disagreements were resolved by discussion with a third reviewer (HG). Authors were contacted by email for missing data and clarifications, in order to achieve completeness of studies.

\section{Data analysis}

The pooled relative risk (RR) was the effect measured of the primary outcome, with $95 \%$ confidence intervals (95\% CI). As the heterogeneity was considerable, the pooled RR was calculated based on a random effect model, according to the 'standard approach' ${ }^{18}$ Statistical heterogeneity was assessed with the $I^{2}$ statistic. ${ }^{19}$

For secondary outcomes, dichotomous data was calculated using RR and continuous data using the standardized mean difference, with 95\% CI and a random effect model.

Included trials were characterized with descriptive statistics (percentages and mean). We performed analyses with Stata $13^{\circledR}$ and RevMan $5.1^{\circledR}$.

\section{Quality assessment}

Every study was independently evaluated for 'risk of bias' by two reviewers (MR and NT), considering the domains and criteria suggested by the Cochrane Handbook v.5.1.0.. ${ }^{12}$ Disagreements were discussed and resolved with a third reviewer (HG).

\section{Other analysis}

Subgroup analysis, publication bias and sensitivity analysis were not possible due to the limited number of trials in the meta-analysis.

\section{RESULTS}

The literature search yielded 104 titles and abstracts, and after eligibility criteria, 7 trials entered to systematic review and meta-analysis (Figure 1). Characteristics of included trials are detailed in table 1 . There were a total of 324 participants with PR, 159 on acyclovir and 165 controls. All studies had two arms, acyclovir and control groups. Only two studies used low doses of acyclovir $(400 \mathrm{mg}$ 5 times daily), while the rest used high doses (800mg 5 times daily). ${ }^{20,21}$ Almost all of the studies had an intervention of seven days, except one (10 days). ${ }^{22}$ All studies include male and females patients with a mean age of 26.9 years old.

\section{Main outcome analysis}

324 participants entered to the meta-analysis, 159 were allocated to acyclovir, and 165 to control. Studies failed to report reasons for dropouts.

\section{Comparison: Acyclovir vs. Placebo (two trials)}

Acyclovir was superior to placebo after one week of treatment (RR 5.72, 95\%CI 2.36-13.88, $\mathrm{I}^{2}=0 \%$ ); however, there was no difference at two weeks (RR 6.08, 95\%CI 0.94-39.36, $\mathrm{I}^{2}=85 \%$ ) (Figure 2). ${ }^{10,23}$

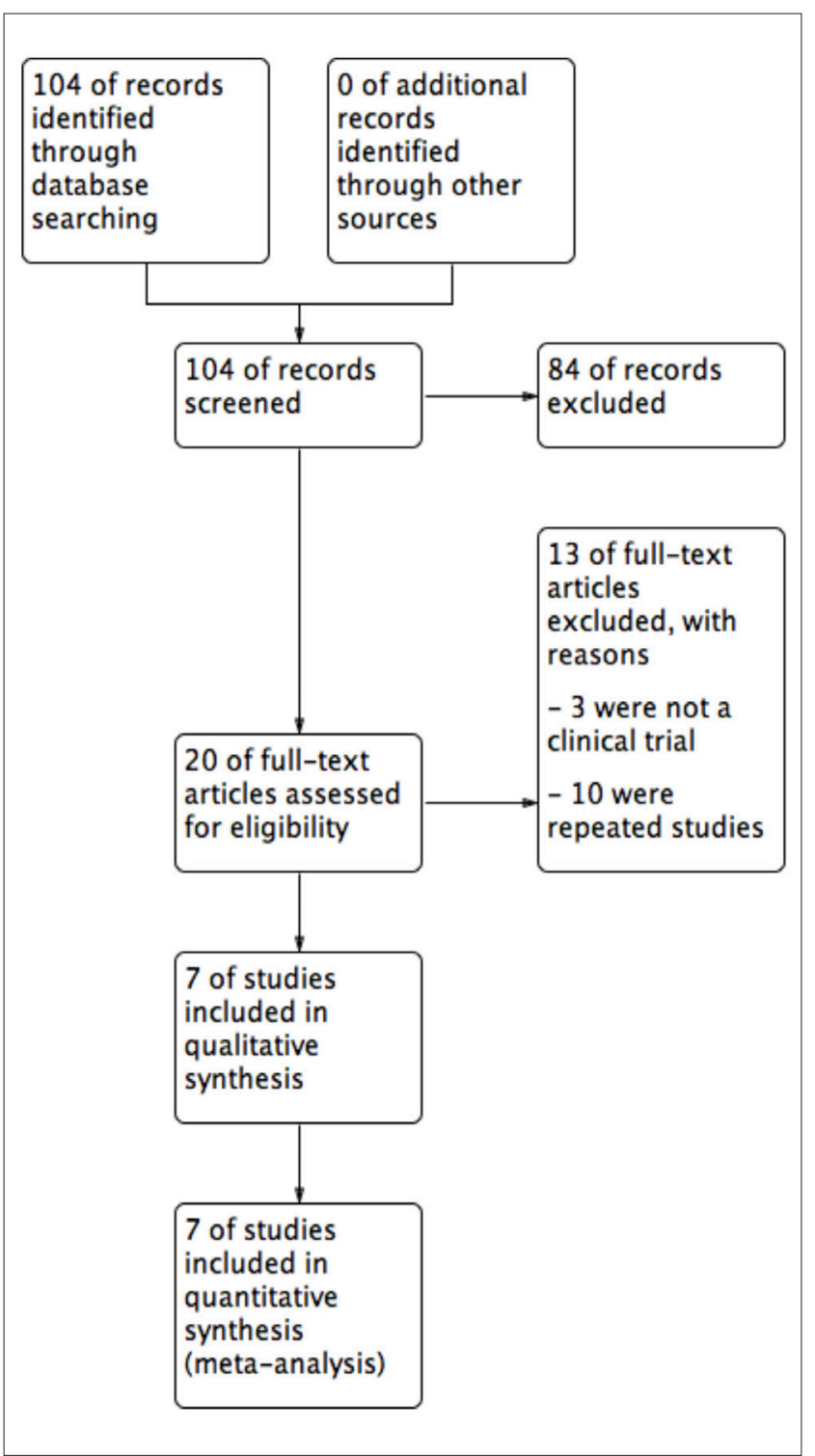

FIGURE 1: Flow diagram of studies included in the meta-analysis

\section{Comparison: Acyclovir vs. antibiotic (one trial)}

Acyclovir was similar to erythromycin after two weeks, but superior after four (RR 8.0 95\% CI 1.13-56.33) and eight (RR 2.16 95\% CI 1.13-4.15) weeks of treatment. ${ }^{22}$

Comparison: Acyclovir vs. observation (one trial)

Acyclovir was superior to observation after one (RR 3.02 95\%CI 1.13-8.08), two (RR 2.92 95\%CI 1.50-5.66) and four (RR 1.51 $95 \%$ CI 1.10-2.08) weeks of treatment. ${ }^{21}$ (two trials)

Comparison: Combined therapy vs. symptomatic treatment

Combined treatment was not significantly superior after two (one study (23): RR 3.11 95\%CI 0.93-10.39), four (two studies $(19,23)$ : RR $1.4695 \%$ CI 0.93-2.29, $\left.\mathrm{I}^{2}=69 \%\right)$ and eight (one study (23): RR 0.98 95\%CI 0.86-1.12) weeks of treatment (Figure 3). ${ }^{24}$ 


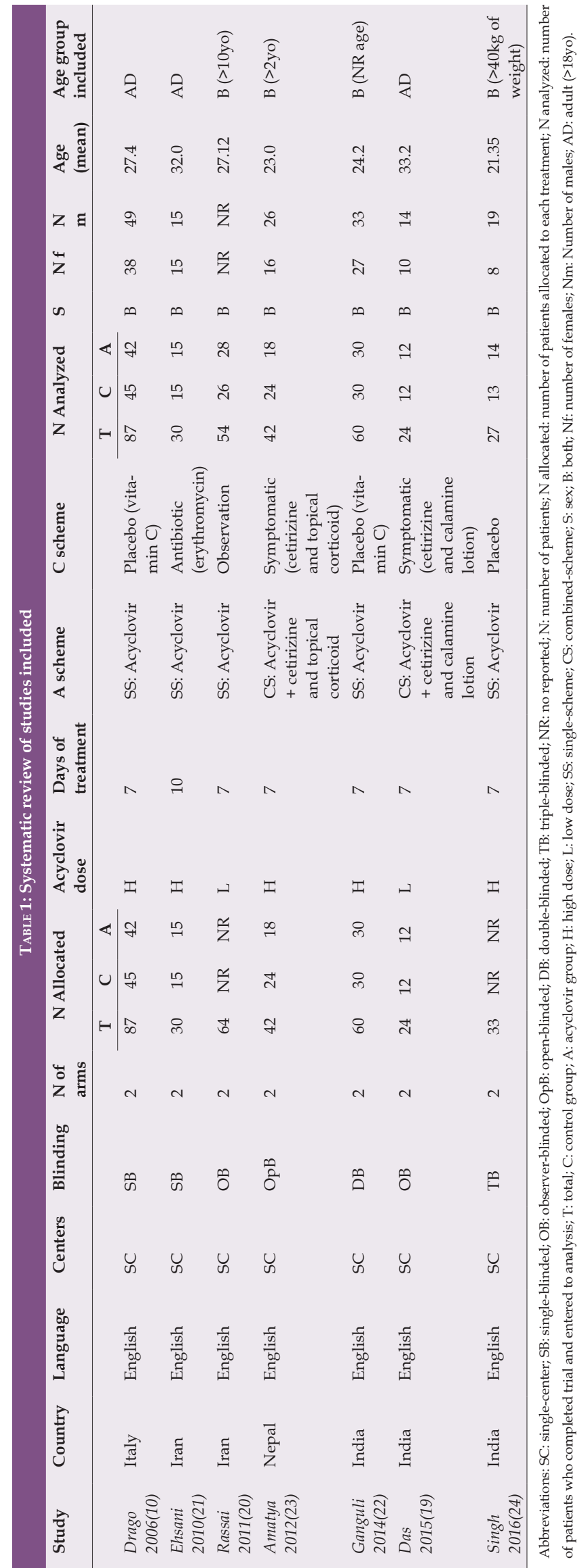

\section{Secondary outcome analysis}

Outcome: Cessation of new lesions

Comparison: Acyclovir vs. Placebo (two trials)

Acyclovir was not significantly superior after one (RR 4.85 95\% CI 0.16-145.64, $\mathrm{I}^{2}=83 \%$ ) and two (RR 1.42 95\%CI 0.73-2.75, $\mathrm{I}^{2}=92 \%$ ) weeks of treatment (Figure 4$) .{ }^{10,23}$ (one trial)

Comparison: Combined therapy vs. symptomatic treatment

Combined therapy was not significantly superior after one week of treatment (RR 1.67, 95\% CI 0.85-3.426). ${ }^{20}$

Outcome: Decrease of symptoms

Comparison: Acyclovir vs. Placebo (one trial)

Acyclovir was not superior to decrease symptoms (e.g. fatigue, headache, sore throat, difficulty in concentrating, irritability, insomnia, and nausea) after one week (RR 12.75, 95\% CI 0.78207.32). ${ }^{10}$ Nevertheless, it was superior at two (RR 11.79 , 95\% CI 1.7380.15 ) weeks of treatment (Figure 5).

\section{Comparison: Acyclovir vs. antibiotic (one trial)}

Acyclovir was not significantly superior to erythromycin to decrease pruritus after two (RR 13.22, 95\% CI 0.91-192.02), four (RR $1.47,95 \%$ CI $0.83-2.61$ ) and eight (RR 1.20, 95\% CI 0.79-1.83) weeks of treatment. ${ }^{22}$ (one trial)

Comparison: Combined therapy vs. symptomatic treatment

Combined therapy decreased significantly pruritus after one (SMD -1.31 95\%CI -2.19, -0.41), two (SMD -1.61 95\%CI -2.53, -0.68 ), three (SMD $-1.2595 \% \mathrm{CI}-2.13,-0.27$ ) and four (SMD -1.21 $95 \%$ CI $-2.10,-0.34$ ) weeks of treatment. ${ }^{20}$

\section{Outcome: duration of disease}

Comparison: Acyclovir vs. Placebo (two trials)

Acyclovir was not superior (SMD -0.04 95\%CI -1.47, 1.38) (Figure 6). ${ }^{10,25}$

The rest of analyses are not presented, as there was not data available.

\section{DISCUSSION}

Regression of lesions

This is the first meta-analysis that evaluates the efficacy of acyclovir for PR. We found that acyclovir was superior to placebo for PR at week 1 for achieving regression of lesions; but when combined with symptomatic treatment, acyclovir was not superior to control at week 4 (Figures 2 and 3). Several studies have linked PR to HHV-6 and 7 as main etiology. ${ }^{5,7}$ However, studies are not conclusive yet, as some have proposed other etiologies, such as other viruses (e.g. HHV-8, cytomegalovirus, Epstein-Barr virus, etc.), association with bacterial and fungal infections, and even immunological origin. ${ }^{26}$ We believe that most of the patients responded to the antiviral when compared to placebo due to the involvement of HHV-6 and 7 (Figure 2). Still, some authors have questioned acyclovir efficacy against $\mathrm{HHV}-7$, as it lacks the thymidine kinase gene. ${ }^{27}$ Nevertheless, efficacy of the antiviral is supported by the results of Watanabe et al. They found systemic active infection of both HHV-6 and 7 at the same time in PR patients, and HHV-6 does respond to acyclovir. ${ }^{28}$ 


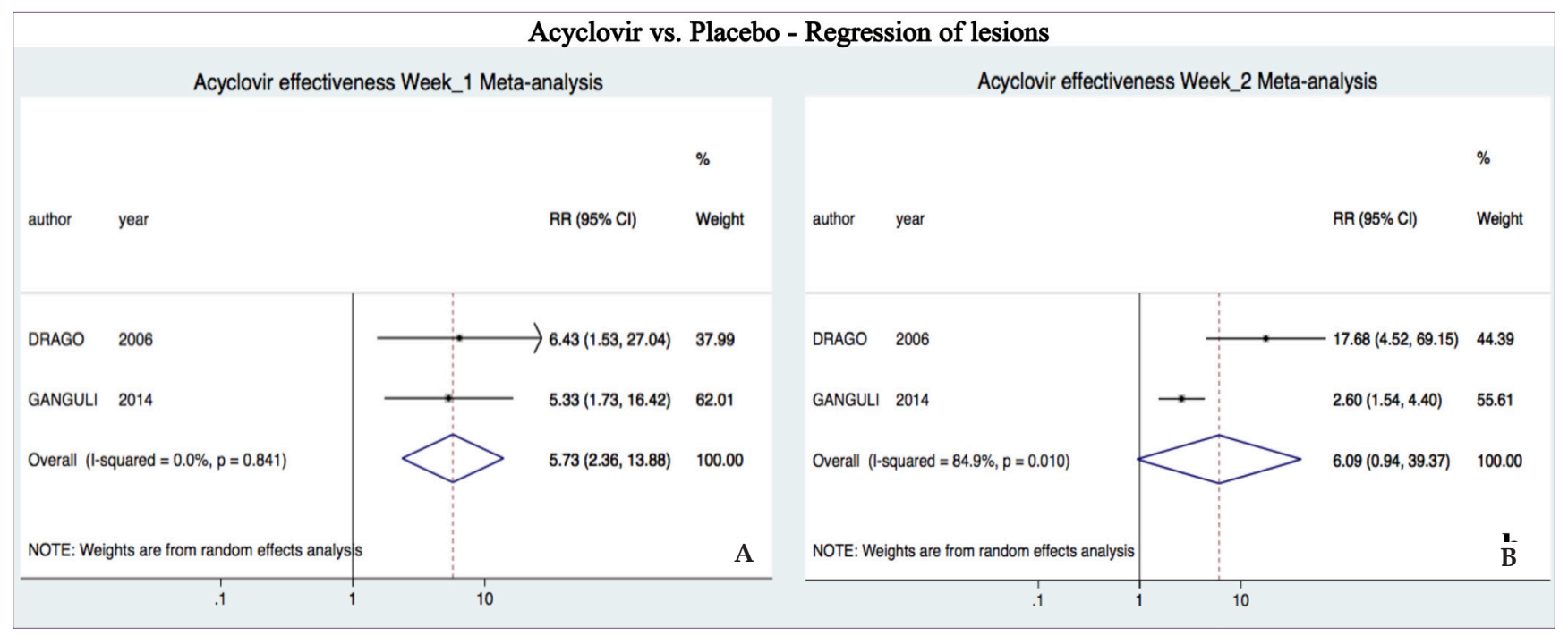

FIGURE 2: Forest plots of meta-analysis for the main outcome (regression of lesions) after one $\mathbf{A}$ and two weeks of treatment $\mathbf{B}$. Trials included compared acyclovir vs. placebo

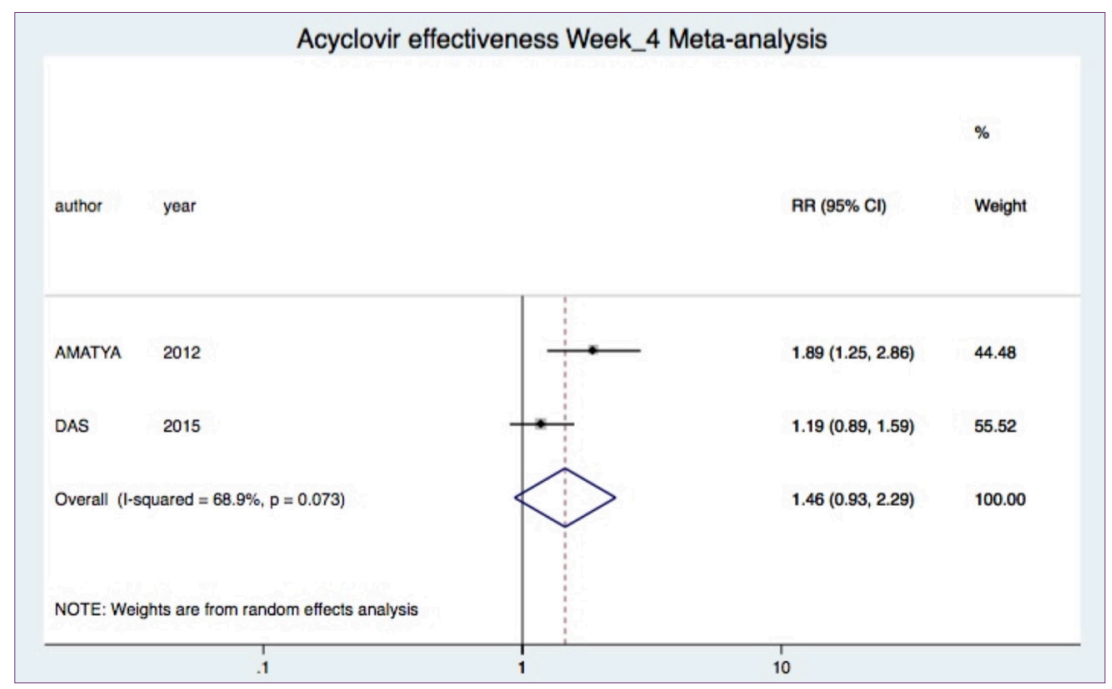

Figure 3: Forest plots of meta-analysis for the main outcome (regression of lesions) after four weeks of treatment. Trials included compared combined therapy vs. symptomatic treatment alone. Combined RR was obtained only for comparison at week 4
When acyclovir was compared with symptomatic treatment, this superiority for regression of lesions was apparently reduced (Figure 3). ${ }^{20,24}$ Thus, symptomatic treatment has an important role for the control of lesions in PR. Guidelines support the use of symptomatic treatment for PR with mild to moderate symptoms. ${ }^{9,11}$ However, a prior meta-analysis found inadequate evidence for efficacy of most symptomatic treatments. ${ }^{17}$ Only a single study showed that oral antihistamine alone was superior than the combination of oral antihistamine and steroids in clearing the PR rash within two weeks. ${ }^{29}$ The symptomatic treatment used in the studies included was oral antihistamine plus topical calamine or corticoid. We suggest that oral antihistamines should be included as primary symptomatic treatment, in association with topical steroids or calamine lotion. ${ }^{30}$

\section{Control of symptoms}

One study ${ }^{10}$ demonstrated that acyclovir was superior to placebo for decreasing symptoms at week 1 and 2 (Figure 5); another study showed the significant decrease of itching from week 1 to 4 with combined treatment. ${ }^{20}$ It is important to underline that other reports had elucidated the anti-pruritic effect of acyclovir in PR. ${ }^{9}$ Other treatments, erythromycin and phototherapy, have been also proposed for pruritic management in PR with controversial results. ${ }^{31}$ Most of the guidelines suggest that, as PR is a self-limited illness, most patients do not need to be treated. Other authors propose that patients with recalcitrant symptoms, such as pruritus, are the exception: they may benefit from treatment with macrolides, phototherapy and antivirals. ${ }^{9}$

When erythromycin is compared to placebo for PR, 3 out of 4 patients on interventional group cleared completely within 2 


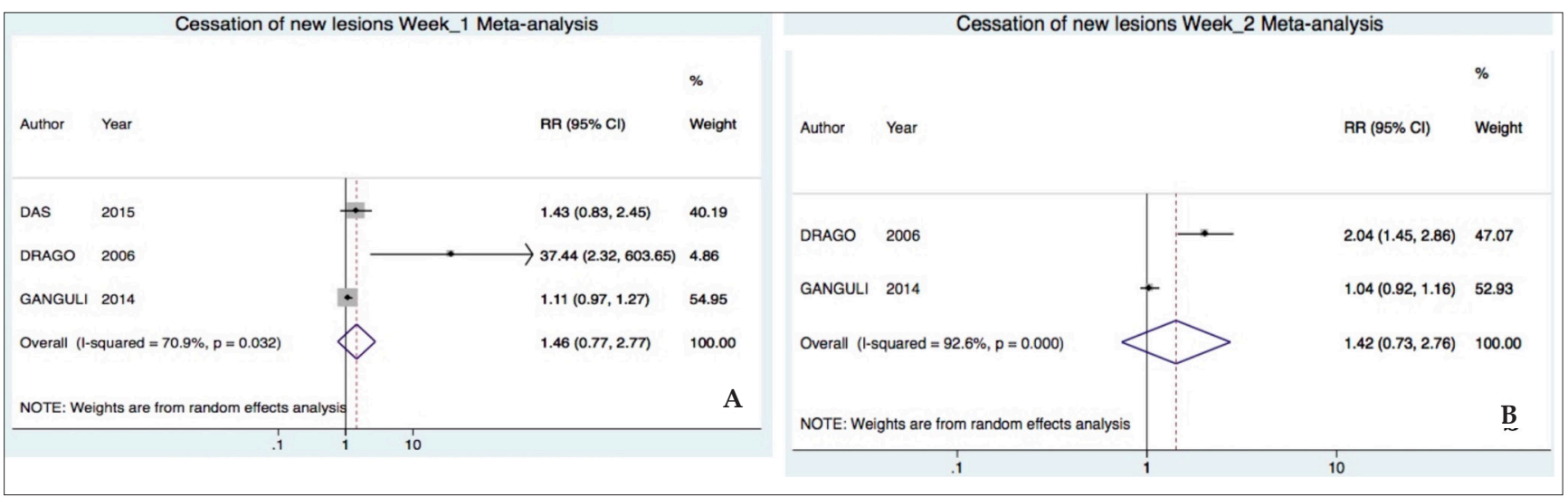

Figure 4: Forest plot of the meta-analysis for secondary outcome cessation of lesions after one A and two B weeks of treatment. Trials included compared acyclovir vs. placebo

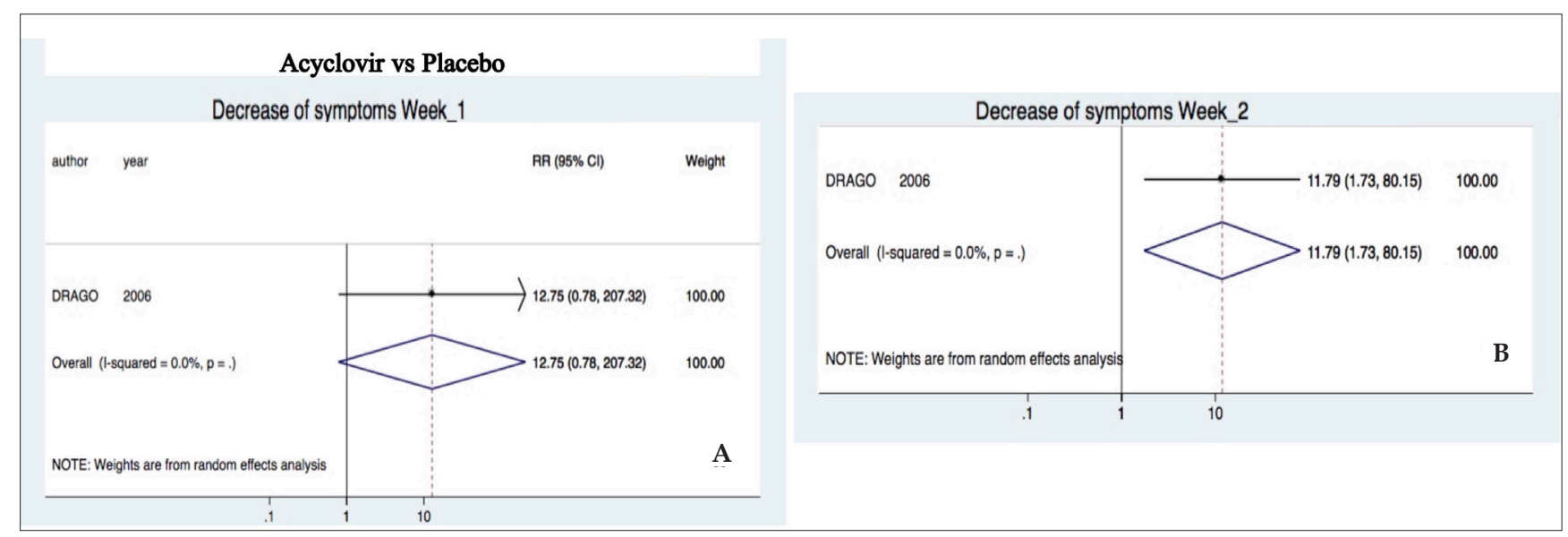

Figure 5: Comparative diagram for decrease of symptoms analysis after one A and two B weeks of treatment. Trials included compared acyclovir vs. placebo

weeks. ${ }^{32}$ Nevertheless, this study had methodological deficiencies in randomization, allocation concealment and analysis, with the adverse events inherent to macrolides. ${ }^{33}$ Other studies suggested immunomodulatory and anti-inflammatory effects of macrolides in PR due to Chlamydia, Legionella and Mycoplasma involvement, but not assertively. ${ }^{34-36}$ Clinical trials have shown that efficacy of macrolides, such as erythromycin, clarithromycin and azithromycin has not been proven. ${ }^{36-38}$ One study compared acyclovir vs. erythromycin and showed that the antiviral was significantly superior for regression of lesions, but not significant for decrease of symptoms. ${ }^{22}$ Therefore, we recommend the use of antiviral over macrolides supported by the possible viral etiology of PR. ${ }^{39}$

Phototherapy with narrowband UVB and UVA1 has been proposed as methods to control PR severity and pruritus. ${ }^{40,41}$ Other studies have proven their efficacy in decreasing severity but failed to change itch and course of the disease. ${ }^{42,43}$ There is inadequate evidence for phototherapy, and sessions are given thrice a week for at least one month. ${ }^{27}$ Although it might be efficient, it may not be feasible and accessible for all patients. ${ }^{31}$

Based on the results, we think that symptomatic treatment (oral antihistamines and topical steroids/calamine) may be considered as baseline treatment for PR, and the early addition of acyclovir for pruritus control, especially in extensive, relapsing or persistent disease. ${ }^{11,17,44}$

\section{Other outcomes}

Acyclovir was not superior to placebo, neither combined to symptomatic treatment in cessation of new lesions at week 1 and 2 
(Figure 4). In addition, acyclovir failed to decrease significantly the duration of PR compared to placebo. However, studies that entered into the meta-analysis had opposed results independently (Figure 6). ${ }^{10,25}$

Clinical trials included were not enough to distinguish efficacy of children from adult population. Drago et al. have found different characteristics between them. ${ }^{45}$ Children have less prevalence of systemic symptoms and less average duration of exanthema. Therefore, we believed that acyclovir in children may not be a reasonable alternative for $\mathrm{PR}$, until trials demonstrate efficacy in this population.

\section{Adverse events}

Adverse events were reported in both acyclovir and control groups (Table 2). In an observer-blinded trial, 8 patients on acyclovir and antihistamines reported increased sleep, headache, nausea, vomiting and dysgeusia. ${ }^{20}$ These adverse events, although they are expected in patients on acyclovir, were not reported in the rest of trials. ${ }^{46} \mathrm{~A}$ triple-blinded trial reported abdominal pain and diarrhea in placebo group, possibly due to nocebo effect. ${ }^{25,47}$

\section{Risk of bias}

Unclear risk of bias was prevalent for allocation concealment (selection bias) and incomplete outcome data (attrition bias) (Figure 7A). The rest of domains had in general low risk of bias. Drago et al, ${ }^{10}$ did not perform randomization neither allocation concealment. Two trials failed to blind participants from treatment received (Figure 7B)..$^{20,24}$

\section{Limitations}

We faced several limitations. First, studies did not evaluate all outcomes and they differed in temporality. Therefore, meta-analysis was not possible for all outcomes and weeks of the follow-up.
Second, some studies had high risk of bias in randomization and allocation concealment. However, we decided to include all trials since they represent interventional studies with similar established outcomes. Finally, there were few trials for each comparison, thus, subgroup analysis was not possible.

TABLE 2: Systematic review of adverse events

\begin{tabular}{|c|c|c|c|c|c|}
\hline \multirow[t]{2}{*}{ Study } & \multicolumn{3}{|c|}{$\mathrm{N}$ of adverse event } & \multicolumn{2}{|c|}{ List of adverse events } \\
\hline & $\mathbf{T}$ & $\mathrm{C}$ & A & $\mathrm{C}$ & A \\
\hline $\begin{array}{l}\text { Drago } \\
2006\end{array}$ & NR & NR & NR & NR & NR \\
\hline $\begin{array}{l}\text { Ehsani } \\
2010\end{array}$ & NR & NR & NR & NR & NR \\
\hline $\begin{array}{l}\text { Rassai } \\
2011\end{array}$ & NR & NR & NR & NR & NR \\
\hline $\begin{array}{l}\text { Amatya } \\
2012\end{array}$ & 8 & 8 & 0 & Dyspepsia & None \\
\hline $\begin{array}{l}\text { Ganguli } \\
2014\end{array}$ & NR & NR & NR & NR & NR \\
\hline $\begin{array}{l}\text { Das } \\
2015\end{array}$ & 9 & 1 & 8 & $\begin{array}{l}\text { Increased } \\
\text { sleep }\end{array}$ & $\begin{array}{l}\text { Increased sleep, } \\
\text { headache, nau- } \\
\text { sea, vomiting, } \\
\text { dysgeusia }\end{array}$ \\
\hline $\begin{array}{l}\text { Singh } \\
2016\end{array}$ & 1 & 1 & 0 & $\begin{array}{l}\text { Abdominal } \\
\text { pain and } \\
\text { diarrhea }\end{array}$ & None \\
\hline
\end{tabular}

Abbreviations: N: number; T: total; C: control group; A: acyclovir group; NR: no reported

\section{Duration of disease Meta-analysis}

Author Year
$\operatorname{SMD}(95 \% \mathrm{Cl})$

Weight

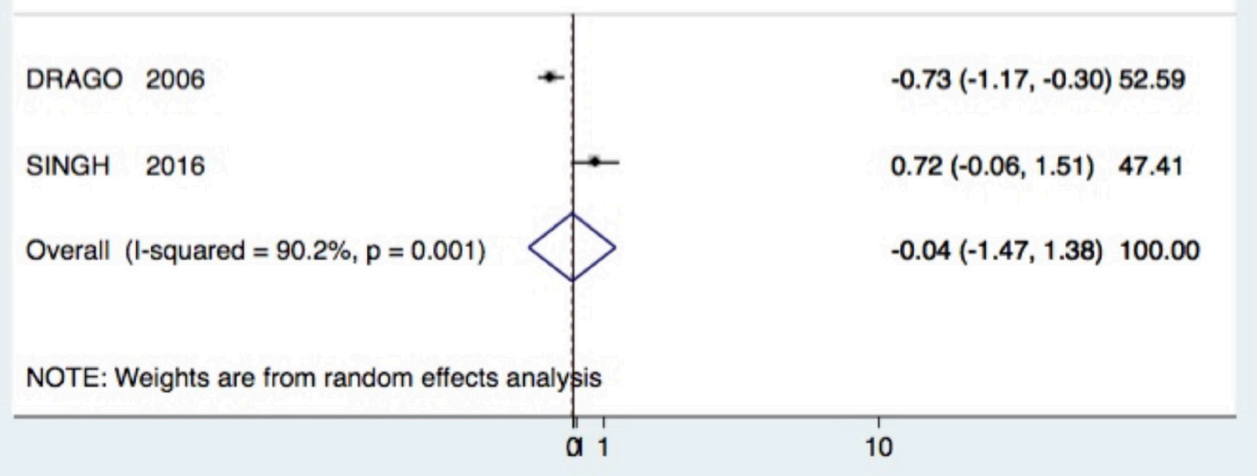

Figure 6: Forest plot of the meta-analysis for secondary outcome duration of disease. Trials included compared acyclovir vs. placebo 


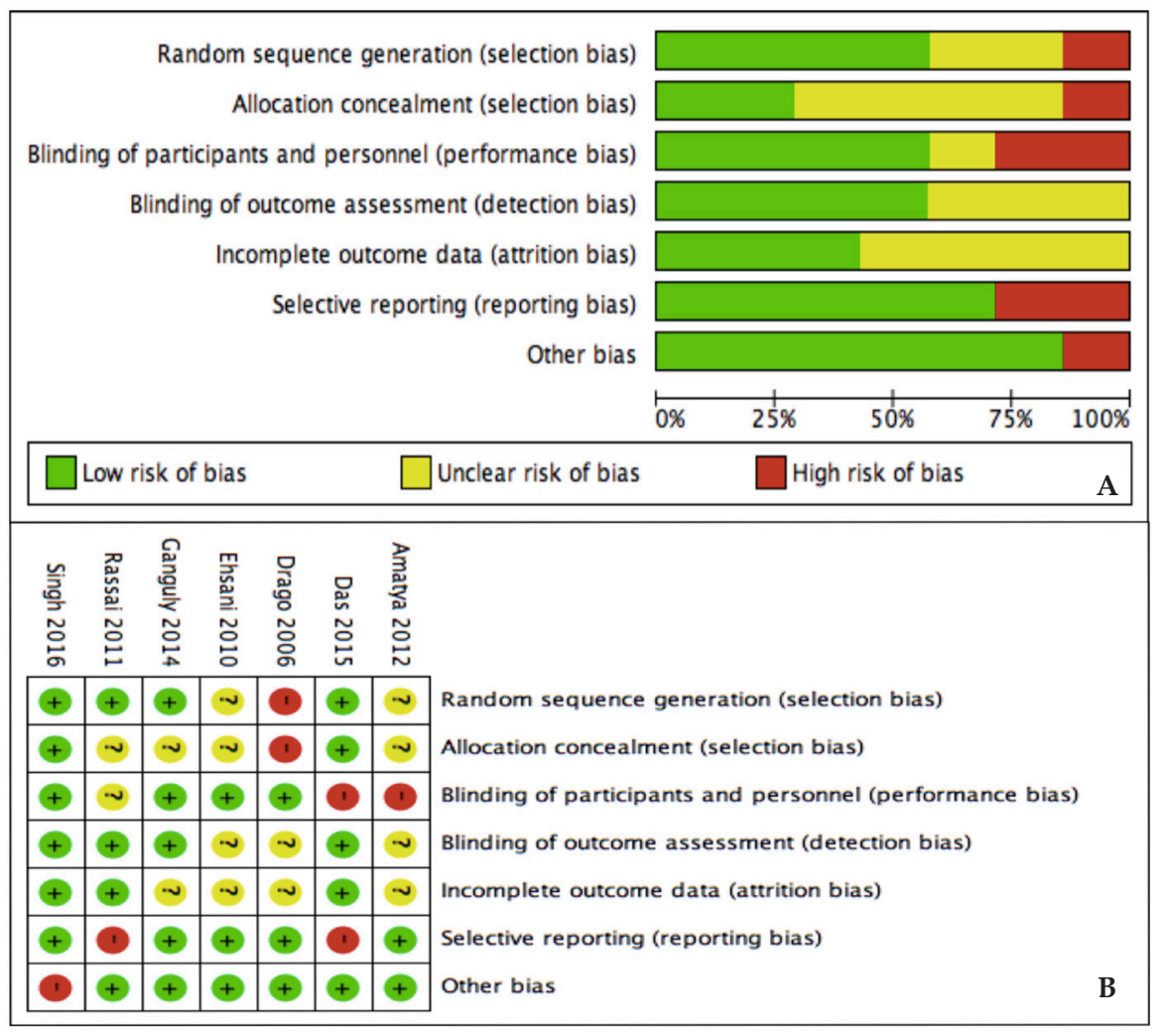

Figure 7: Risk of bias analysis. A. Between studies; B. Within studies

\section{CONCLUSION}

Acyclovir was superior to placebo for regression of lesions of PR. However, combined therapy was not superior to symptomatic treatment for control of lesions. Individual trials indicated that acyclovir was superior to placebo and symptomatic treatment for the control of symptoms and pruritus. Finally, acyclovir failed to prevent new lesions and to shorten the duration of the disease when compared to placebo.

Our results reflect that symptomatic treatment, as oral antihistamines and topical steroids/calamine, plays an important role for PR in the regression of lesions, given that there was no difference when compared to combined treatment. We recommend the addition of acyclovir for pruritus control and it might be justified also for severe symptoms, within four weeks of the onset of PR. ${ }^{6}$

More trials need to be conducted in order to prove the efficacy of acyclovir for PR. We encourage researchers to follow similar designs and to adopt similar outcomes to unify criteria for future meta-analysis. Interventions should include acyclovir plus symptomatic treatment in therapy group. In addition, report of trials should account for randomization, allocation concealment and dropouts in order to increase quality of studies.

\section{REFERENCES}

1. Parsons JM. Pityriasis rosea update: 1986. J Am Acad Dermatol. 1986;15:159-67.

2. Drago F, Ciccarese G, Rebora A, Broccolo F, Parodi A. Pityriasis rosea: a comprehensive classification. Dermatology. 2016;232:431-7.

3. Kilinc F, Akbas A, Sener S, Aktas A. Atypical pityriasis rosea: clinical evaluation of 27 patients. Cutan Ocul Toxicol. 2017;36:157-162.

4. Drago F, Broccolo F, Rebora A. Pityriasis rosea: an update with a critical appraisal of its possible herpesviral etiology. J Am Acad Dermatol. 2009;61:303-18.

5. Canpolat Kirac B, Adisen E, Bozdayi G, Yucel A, Fidan I, Aksakal N, et al. The role of human herpesvirus 6, human herpesvirus 7, Epstein-Barr virus and cytomegalovirus in the aetiology of pityriasis rosea. J Eur Acad Dermatol Venereol. 2009:23:16-21.

6. Mahajan K, Relhan V, Relhan AK, Garg VK. Pityriasis Rosea: An update on etiopathogenesis and management of difficult aspects. Indian J Dermatol. 2016;61:375-84
7. Drago F, Ranieri E, Malaguti F, Battifoglio ML, Losi E, Rebora A. Human herpesvirus 7 in patients with pityriasis rosea. Electron microscopy investigations and polymerase chain reaction in mononuclear cells, plasma and skin. Dermatology. 1997; 195:374-8.

8. Chuh A, Chan H, Zawar V. Pityriasis rosea--evidence for and against an infectious aetiology. Epidemiol Infect. 2004;132:381-90.

9. Chuh A, Zawar V, Sciallis G, Kempf W. A position statement on the management of patients with pityriasis rosea. J Eur Acad Dermatol Venereol. 2016;30:1670-81.

10. Drago F, Vecchio F, Rebora A. Use of high-dose acyclovir in pityriasis rosea. J Am Acad Dermatol. 2006;54:82-5.

11. Ciccarese G, Drago F. Is a treatment for pityriasis rosea really needed? Indian Dermatol Online J. 2016;7:435. 
12. Handbook.cochrane.org [Internet]. Higgins J, Green S. Cochrane Handbook for Systematic Reviews of Interventions: The Cochrane Collaboration; 2011 [cited 2016 Nov 10]. Available from: http://handbook.cochrane.org.

13. Liberati A, Altman DG, Tetzlaff J, Mulrow C, Gotzsche PC, Ioannidis JP, et al. The PRISMA statement for reporting systematic reviews and meta-analyses of studies that evaluate healthcare interventions: explanation and elaboration. BMJ. 2009;339:b2700.

14. Moher D, Liberati A, Tetzlaff J, Altman DG, PRISMA Group. Preferred Reporting Items for Systematic Reviews and Meta-Analyses: The PRISMA Statement. PLoS Med. 2009;6:e1000097.

15. Frenkel N, Schirmer EC, Wyatt LS, Katsafanas G, Roffman E, Danovich RM, et al. Isolation of a new herpesvirus from human CD4+ T cells. Proc Natl Acad Sci U S A. 1990;87:748-52.

16. Yamanishi K, Okuno T. [New human herpesvirus, human herpes virus-6]. Nihon Rinsho. 1989:47:285-9.

17. Chuh AA, Dofitas BL, Comisel GG, Reveiz L, Sharma V, Garner SE, et al. Interventions for pityriasis rosea. Cochrane Database Syst Rev. 2007:CD005068.

18. DerSimonian R, Laird N. Meta-analysis in clinical trials revisited. Contemp Clin Trials. 2015;45:139-45.

19. Higgins JP, Thompson SG, Deeks JJ, Altman DG. Measuring inconsistency in meta-analyses. BMJ. 2003;327:557-60.

20. Das A, Sil A, Das NK, Roy K, Das AK, Bandyopadhyay D. Acyclovir in pityriasis rosea: An observer-blind, randomized controlled trial of effectiveness, safety and tolerability. Indian Dermatol Online J. 2015;6:181-4.

21. Rassai S, Feily A, Sina N, Abtahian S. Low dose of acyclovir may be an effective treatment against pityriasis rosea: a random investigator-blind clinical trial on 64 patients. J Eur Acad Dermatol Venereol. 2011;25:24-6.

22. Ehsani A, Esmaily N, Noormohammadpour P, Toosi S, Hosseinpour A, Hosseini $\mathrm{M}$, et al. The comparison between the efficacy of high dose acyclovir and erythromycin on the period and signs of pitiriasis rosea. Indian $\mathrm{J}$ Dermatol. 2010:55:246-8.

23. Ganguly S. A randomized, double-blind, placebo-controlled study of efficacy of oral acyclovir in the treatment of pityriasis rosea. J Clin Diagn Res. 2014;8:YC01-4.

24. Amatya A, Rajouria EA, Karn DK. Comparative study of effectiveness of oral acyclovir with oral erythromycin in the treatment of pityriasis rosea. Kathmandu Univ Med J (KUMJ). 2012;10:57-61.

25. Singh S, Anurag, Tiwary NK. Acyclovir is not effective in pityriasis rosea: results of a randomized, triple-blind, placebo-controlled trial. Indian J Dermatol Venereol Leprol. 2016;82:505-9.

26. Guarneri F, Cannavo SP, Minciullo PL, Gangemi S. Pityriasis rosea of Gibert: immunological aspects. J Eur Acad Dermatol Venereol. 2015;29:21-5.

27. Chuh A. Narrow band UVB phototherapy and oral acyclovir for pityriasis rosea. Photodermatol Photoimmunol Photomed. 2004;20:64-5.

28. Watanabe T, Kawamura T, Jacob SE, Aquilino EA, Orenstein JM, Black JB, et al. Pityriasis rosea is associated with systemic active infection with both human herpesvirus-7 and human herpesvirus-6. J Invest Dermatol. 2002;119:793-7.
29. Lazaro-Medina A, Villena-Amurao C, Dy-Chua NS, Sit-Toledo MSW, Villanueva B. A clinicohistopathologic study of a randomized double-blind clinical trial using oral dexchlorpheniramine $4 \mathrm{mg}$, betamethasone $500 \mathrm{mcg}$ and betamethasone $250 \mathrm{mcg}$ with dexchlorpheniramine $2 \mathrm{mg}$ in the treatment of pityriasis rosea: a preliminary report. Journal of the Philippine Dermatological Society. 1996;5:3-7.

30. Stulberg DL, Wolfrey J. Pityriasis rosea. Am Fam Physician. 2004;69:87-91.

31. Gambichler T, Terras S, Kreuter A. Treatment regimens, protocols, dosage, and indications for UVA1 phototherapy: facts and controversies. Clin Dermatol. 2013:31:438-54.

32. Sharma PK, Yadav TP, Gautam RK, Taneja N, Satyanarayana L. Erythromycin in pityriasis rosea: a double-blind, placebo-controlled clinical trial. J Am Acad Dermatol. 2000;42:241-4.

33. Bigby M. A remarkable result of a double-masked, placebo-controlled trial of erythromycin in the treatment of pityriasis rosea. Arch Dermatol. 2000;136:775-6.

34. Terraneo L, Lava SA, Camozzi P, Zgraggen L, Simonetti GD, Bianchetti MG, et al. Unusual eruptions associated with Mycoplasma pneumoniae respiratory infections: review of the literature. Dermatology. 2015;231:152-7.

35. Chuh AA, Chan HH. Prospective case-control study of chlamydia, legionella and mycoplasma infections in patients with pityriasis rosea. Eur $\mathrm{J}$ Dermatol. 2002;12:170-3

36. Rasi A, Tajziehchi L, Savabi-Nasab S. Oral erythromycin is ineffective in the treatment of pityriasis rosea. J Drugs Dermatol. 2008;7:35-8.

37. Pandhi D, Singal A, Verma P, Sharma R. The efficacy of azithromycin in pityriasis rosea: a randomized, double-blind, placebo-controlled trial. Indian J Dermatol Venereol Leprol. 2014;80:36-40.

38. Ahmed N, Iftikhar N, Bashir U, Rizvi SD, Sheikh Zl, Manzur A. Efficacy of clarithromycin in pityriasis rosea. J Coll Physicians Surg Pak. 2014;24:802-5.

39. Drago F, Ciccarese G, Rebora A, Parodi A. The efficacy of macrolides and acyclovir in pityriasis rosea. Indian J Dermatol Venereol Leprol. 2015;81:56.

40. Jairath V, Mohan M, Jindal N, Gogna P, Syrty C, Monnappa PM, et al. Narrowband UVB phototherapy in pityriasis rosea. Indian Dermatol Online J. 2015;6:326-9.

41. Lim SH, Kim SM, Oh BH, Ko JH, Lee YW, Choe YB, et al. Low-dose ultraviolet A1 phototherapy for treating pityriasis rosea. Ann Dermatol. 2009;21:230-6.

42. Valkova S, Trashlieva M, Christova P. UVB phototherapy for pityriasis rosea. J Eur Acad Dermatol Venereol. 2004:18:111-2.

43. Leenutaphong V, Jiamton S. UVB phototherapy for pityriasis rosea: a bilateral comparison study. J Am Acad Dermatol. 1995;33:996-9.

44. Drago F, Ciccarese G, Rebora A, Parodi A. Relapsing pityriasis rosea. Dermatology. 2014;229:316-8.

45. Drago F, Ciccarese G, Broccolo F, Cozzani E, Parodi A. Pityriasis rosea in children: clinical features and laboratory investigations. Dermatology. 2015;231:9-14.

46. Heslop R, Roberts H, Flower D, Jordan V. Interventions for men and women with their first episode of genital herpes. Cochrane Database Syst Rev. 2016:CD010684.

47. Rojas-Mirquez JC, Rodriguez-Zuniga MJ, Bonilla-Escobar FJ, Garcia-Perdomo HA, Petkov M, Becerra L, et al. Nocebo effect in randomized clinical trials of antidepressants in children and adolescents: systematic review and metaanalysis. Front Behav Neurosci. 2014;8:375.

\section{AUTHORS'CONTRIBUTIONS}

Milton Rodriguez-Zuniga

(iD) ORCID 0000-0002-9413-2439

Statistical analysis, Approval of the final version of the manuscript, Design and planning of the study, Preparation and writing of the manuscript, Collecting, analysis and interpretation of data, Effective participation in research orientation, Intellectual participation in propaedeutic and/or therapeutic conduct of studied cases, Critical review of the literature, Critical review of the manuscript

Natalie Torres (D) ORCID 0000-0001-8051-6426

Statistical analysis, Approval of the final version of the manuscript, Design and planning of the study, Preparation and writing of the manuscript, Collecting, analysis and interpretation of data, Effective participation in research orientation, Intellectual participation in propaedeutic and/or therapeutic conduct of studied cases, Critical review of the literature, Critical review of the manuscript
Herney Garcia-Perdomo D ORCID 0000-0001-6945-8261

Statistical analysis, Approval of the final version of the manuscript, Design and planning of the study, Preparation and writing of the manuscript, Collecting, analysis and interpretation of data, Effective participation in research orientation, Intellectual participation in propaedeutic and/or therapeutic conduct of studied cases, Critical review of the literature, Critical review of the manuscript

How to cite this article: Rodriguez-Zuniga MJM, Torres N, Garcia-Perdomo H. Effectiveness of acyclovir in the treatment of pityriasis rosea. A systematic review and meta-analysis. An Bras Dermatol. 2018;93(5):686-95. 